\title{
Estudo da conformação de substratos cerâmicos por laminação a partir de suspensões concentradas de alumina
}

\section{(Rolling study of ceramic substrates from concentrate alumina suspensions)}

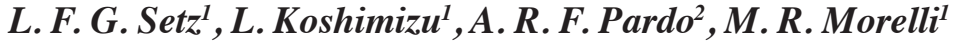 \\ ${ }^{I}$ Departamento de Engenharia de Materiais, Universidade Federal de S. Carlos, \\ Rod. Washington Luís, km 235, S. Carlos, SP 13565-905 \\ ${ }^{2}$ Petróleo Brasileiro S.A., EDISE, Av. Chile, 65, Centro, Rio de Janeiro, RJ 20031-912 \\ lfsetz@yahoo.com.br,arfpardo@yahoo.com.br,laiskoshimizu@yahoo.com.br,morelli@ufscar.br
}

\begin{abstract}
Resumo
A produção de substratos cerâmicos por laminação, ou conformação viscoplástica, é interessante, pois minimiza problemas inerentes ao processamento como a aglomeração dos pós. Quando a preparação das massas a serem conformadas por esta técnica é realizada a partir de suspensões estáveis, estes problemas quase inexistem, possibilitando a obtenção de produtos íntegros com microestrutura homogênea. Neste trabalho é apresentado o comportamento reológico das suspensões concentradas e das massas de alumina contendo diferentes teores do espessante/plastificante hidroxipropil metilcelulose (HPMC) e também o estudo das variáveis de processo envolvidas na conformação por calandragem. Como resultado deste estudo foi possível obter substratos de alumina calandrados densos, utilizando-se uma suspensão concentrada (60\% vol.), estabilizada com $0,02 \%$ de Viscocrete $20 \mathrm{HE}$ e com adição de $1,5 \%$ m. de HPMC.
\end{abstract}

Palavras-chave: reologia, substratos cerâmicos, calandragem.

Abstract

The ceramic substrates production by calendering, or viscous plastic processing, is interesting because inherent problems as a powder agglomeration is minimized. When the ceramic pastes shaping for this technique are produced from stabilized suspensions these problems almost inexist. This work presents the concentrate suspensions and pastes with different hydroxypropyl methyl cellulose (HPMC) thickener content rheological behaviors. The variables involved in calendering shaping were studied, too. The production of dense alumina substrates shaped by calendaring from concentrate suspensions (60 vol.\%), stabilized with 0.02 wt.\% Viscocrete $20 \mathrm{HE}$ and $1.5 \mathrm{wt}$.\% HPMC is possible.

Keywords: rheology, ceramic substrates, calendering.

\section{INTRODUÇÃO}

A conformação plástica envolve a produção de peças a partir de uma mistura de pós e aditivos que proporcionam uma massa plástica coerente e deformável, e também alguma capacidade de manter a sua forma determinada. Estes processos são extensivamente utilizados na fabricação de cerâmicas tradicionais como telhas, vasos, tijolos entre outros. Nas argilas, a plasticidade é conseguida apenas com a adição de uma pequena quantidade de água [1]. Porém, nos sistemas de "óxidos puros", tais como os constituídos por alumina, ítria, zircônia, etc., por não possuírem a característica de plasticidade, é necessária a adição de materiais orgânicos (ligantes e plastificantes) acompanhados de um solvente para proporcionar adequada plasticidade e elasticidade. Geralmente é necessária a adição de 25 a $50 \%$ de material orgânico, em massa, para se conseguir a adequada plasticidade para conformação [2].

O comportamento plástico de uma pasta cerâmica extrudável é controlado por diversos fatores [3]: (i) fração volumétrica, forma e distribuição de tamanho das partículas, (ii) tipo de forças interpartículas dominantes, (iii) química de superfície, (iv) densidade de empacotamento e (v) comportamento reológico da fase líquida. O controle das propriedades da fase líquida (contínua) é crítica para o sucesso do processo. Como regra geral, a pasta deve ser a mais viscosa possível com a mínima taxa de deformação (ou cisalhamento).

A medição e controle da plasticidade são essenciais para alcançar boas condições de fabricação (formas corretas e baixos tempos de processamento). No entanto, a prática comum é empírica, devido ao grande número de variáveis de processo envolvidas e da ausência de meios sensíveis de quantificação para a avaliação das complexas relações entre as características de fluxo e as propriedades dos componentes como extrudados [4]. O comportamento plástico de massas cerâmicas geralmente é previsto por curvas tensão-deformação de compressão $[2,5]$. Por outro lado, tem-se que o comportamento reológico de suspensões 
cerâmicas é bem compreendido, e desta forma, pode se estender estas análises para a compreensão e controle de produção de massas cerâmicas plásticas, a partir de suspensões concentradas, fornecendo subsídios para se obter de forma controlada, ao final do processo, produtos íntegros e adequados à aplicação desejada [6].

A conformação de massas cerâmicas plásticas por laminação, ou calandragem, ou ainda, chamado de processamento viscoplástico (viscous plastic processing of ceramics - VPP) permite a obtenção de corpos cerâmicos em uma faixa de espessura de considerável interesse tecnológico $(0,3 \sim 5,0 \mathrm{~mm})$, bastante adequada à produção de substratos para cerâmicas eletrônicas, revestimentos (pisos e azulejos) e membranas multicamadas, como são as membranas assimétricas utilizadas para suporte de catalisadores e filtros para tratamento de água [7]. O processo de conformação por laminação, ilustrado na Fig. 1 [8], é um dos processos mais utilizados para conformação mecânica de metais, pois proporciona alta produtividade com um controle dimensional, do produto acabado, bastante preciso [9]. Por essas características o processo torna-se interessante também para o processamento cerâmico.

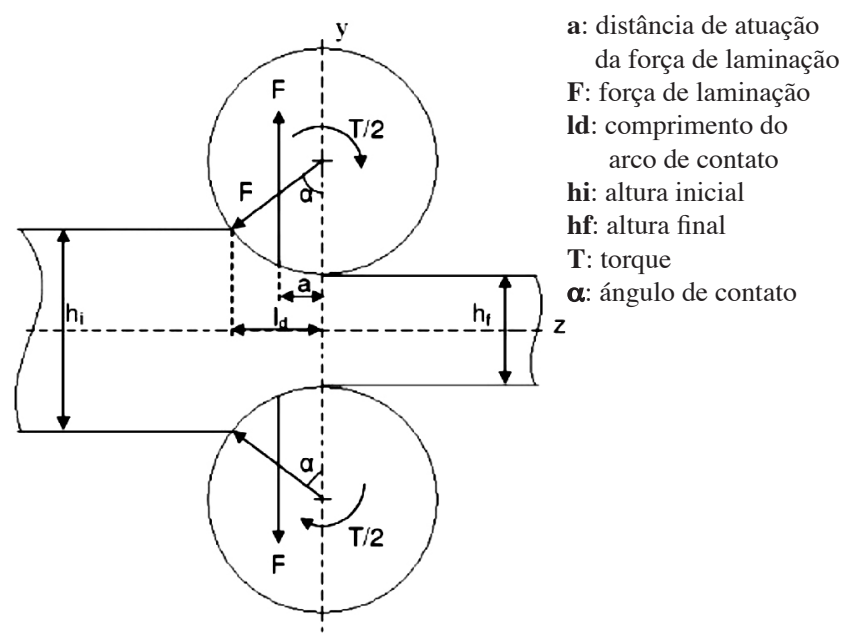

Figura 1: Diagrama do processo de laminação com esforços envolvidos no processo [8].

[Figure 1: Diagram of the process involved in efforts to rolling system [8].]

Os produtos obtidos por VPP oferecem grande resistência e homogeneidade, um bom acabamento da superfície final, além de melhor tenacidade, quando comparados com os produtos convencionais, e a melhora nas propriedades permite a confecção de componentes com formas complexas, peso reduzido e espessura de parede mais fina, sem comprometer o desempenho [10]. O processo é rápido e, geralmente, envolve pouca quantidade de água e aditivos poliméricos $[10,11]$.

Neste âmbito, o objetivo do trabalho é obter e caracterizar substratos cerâmicos densos, preparados a partir de suspensões estáveis com elevado conteúdo de sólidos, adicionados de elementos plastificantes capazes de serem conformados pelo processo de laminação.

\section{MATERIAIS E MÉTODOS}

Para a caracterização reológica das suspensões e massas, e posterior preparação dos substratos, utilizou-se $\alpha-\mathrm{Al}_{2} \mathrm{O}_{3}$ calcinada (A17-NE, Almatis, EUA), com as seguintes características: $\mathrm{D}_{50} 3,34 \mu \mathrm{m}$, área de superfície específica de $2,98 \mathrm{~m}^{2} \cdot \mathrm{g}^{-1}$, densidade de $3,97 \mathrm{~g} \cdot \mathrm{m}^{-3}$ e $99,8 \%$ de pureza. A estabilização das suspensões fez-se utilizando o dispersante Viscocrete 20HE (solução aquosa de poliacrilatos modificados, Silka, Suíça) com variações de concentração de 0,01 a 1,05\% em massa, em relação à alumina, para uma concentração de sólidos fixa de $50 \%$ em volume ( $80 \%$ em massa). O dispersante utilizado é utilizado na indústria de cimentos e de concretos [12] e apresenta como grande atrativo uma configuração tipo "pente", ou seja, uma cadeia polimérica com ramificações que o torna potencialmente mais efetivo na estabilização de suspensões cerâmicas em comparação a outros dispersantes tradicionalmente utilizados, como o ácido cítrico [13], por exemplo.

O comportamento reológico foi avaliado de duas maneiras. A primeira etapa correspondeu à preparação e estudo de suspensões com elevada concentração de sólidos. As suspensões foram preparadas utilizando-se água deionizada e misturadas em agitador mecânico (Ética Equipamentos Científicos S.A., Brasil) com haste tipo hélice por $1 \mathrm{~h}$, e em seguida, medidas em viscosímetro (Haake VT500, Thermo Scientific, Alemanha) utilizando-se como sensor cilindros concêntricos (MV DIN) correspondendo a um sistema de medição do tipo Searle. Variou-se a velocidade de cisalhamento entre 2,5 e $200 \mathrm{~s}^{-1}$, registrandose 10 passos intermediários.

A segunda etapa correspondeu à preparação e caracterização reológica das massas cerâmicas. Às suspensões com alta concentração de sólidos (60\%vol, $\sim 86 \% \mathrm{~m}$.) adicionou-se o aditivo plastificante hidroxipropil metilcelulose 3000 (HPMC 3000, Dow Wolff Cellulosics, EUA) em concentrações de 0,5, 1,0 e 1,5\% em massa, a mistura e homogeneização ocorreu em moinho de facas, semelhante a uma extrusora de rosca. A avaliação do comportamento reológico das massas plásticas produzidas foi executada em um reômetro tipo capilar onde, a massa é pressionada por um pistão que se movimenta com velocidade controlada. Neste equipamento registra-se a força necessária para extrudar a massa por um bocal em função da velocidade de movimentação do pistão. O reômetro apresenta-se acoplado a uma máquina de ensaios universal (5200, Instron, EUA), capaz de controlar a velocidade de descida do pistão e de monitorar a força. O bocal de saída do reômetro possui perfil circular, com diâmetro de $10 \mathrm{~mm}$, suficiente para extrudar massas com alta concentração de sólidos. As velocidades de deslocamento do pistão utilizadas variaram de 40 a $320 \mathrm{~mm} \cdot \mathrm{min}^{-1}$.

As massas produzidas foram conformadas por laminação por rolos a frio (cilindro laminador, Lieme Ind. Metalúrgica Ltda., Brasil) que permitem o controle e a variação da rotação entre 0 e $60 \mathrm{rpm}$ e uma espessura máxima entre os rolos, ajustada manualmente, de $5 \mathrm{~mm}$. As variáveis de 
conformação estudadas foram as rotações de 5,20 e 50 rpm, e a distância entre rolos de 1,5; 3,0 e 4,0 mm. Após conformação os substratos foram secos a $110^{\circ} \mathrm{C}$ por $24 \mathrm{~h}$.

As densidades a verde dos substratos conformados foram determinadas por porosimetria de intrusão de mercúrio (Poresizer 9320, Micromeritics, EUA). Os substratos foram sinterizados a $1600^{\circ} \mathrm{C} / 1 \mathrm{~h}$ em forno elétrico e as densidades finais foram determinadas pelo método de imersão em água baseado no princípio de Arquimedes.

Os substratos sinterizados tiveram seus módulos de resistência à flexão (MRF) determinados por ensaios de flexão em três pontos em máquina de ensaios universal (5200, Instron, EUA), com distância entre apoios de $45 \mathrm{~mm}$.

As microestruturas das superfícies de fratura dos corpos de prova foram analisadas em microscópio eletrônico de varredura (Stereoscan 440, Leica, Alemanha).

\section{RESULTADOS E DISCUSSÃO}

A primeira etapa para preparação de suspensões adequadas, independente do processo de conformação utilizado, consiste em estabilizar a suspensões, seja por meio de ajuste de $\mathrm{pH}$ (estabilização eletrostática), com adição de polímeros (estabilização estérica) ou polieletrólitos (estabilização eletroestérica) [14, 15]. Neste trabalho, ajustou-se a concentração de dispersante em suspensões concentradas, mantidas constante a 50\%vol. $(\sim 80 \% \mathrm{~m})$.

Na Fig. 2 é apresentada a curva de viscosidade em função da concentração do dispersante. A adição de uma pequena concentração do dispersante Viscocrete $(0,01 \%$ em massa) melhora significativamente o comportamento da suspensão quando comparado com a suspensão sem dispersante, reduzindo significativamente sua viscosidade. O incremento da concentração do dispersante reduz a viscosidade da dispersão até $0,02 \% \mathrm{~m}$., permanecendo praticamente

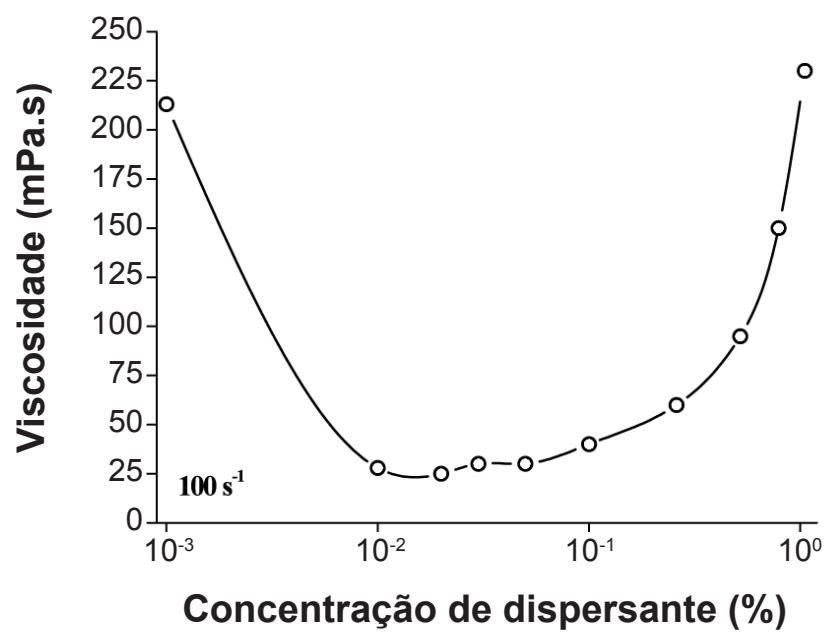

Figura 2: Curva de viscosidade de suspensões de alumina em função da concentração de dispersante (Viscocrete) a uma taxa de cisalhamento constante de $100 \mathrm{~s}^{-1}$.

[Figure 2: Viscosity of alumina suspensions versus dispersant (Viscocrete) concentration curve for a constant shear rate at $100 \mathrm{~s}^{-1}$.] inalterada até adições da ordem de $0,05 \%$ m., a partir da qual a suspensão tem sua viscosidade significativamente elevada. Isto significa que a estabilidade da suspensão é alcançada com adições de $0,02 \% \mathrm{~m}$. de dispersante e que, a partir deste valor, a interação do polímero com as partículas inflige o comportamento inverso ao desejado, fazendo com que ocorra a aglomeração das partículas. Este efeito, possivelmente, tem a contribuição do emaranhamento das ramificações poliméricas do dispersante. A quantidade de Viscocrete necessária, portanto, à estabilização de suspensões concentradas de alumina, é de $0,02 \% \mathrm{~m}$., ou seja, 10 vezes inferior a quantidade usual de ácido cítrico $(0,2 \% \mathrm{~m})$ [13].

A variação da viscosidade em função do aumento do teor de sólidos das suspensões é ilustrada na Fig. 3. Considerando-se que para o processo de laminação suspensões com elevados valores de viscosidade são inadequadas e baseando-se nos resultados apresentados na Fig. 3, selecionou-se para a preparação de substratos, suspensões com $60 \%$ de sólidos em volume, uma vez que para o processo de laminação dos substratos ainda é adicionado o aditivo espessante/plastificante, HPMC.

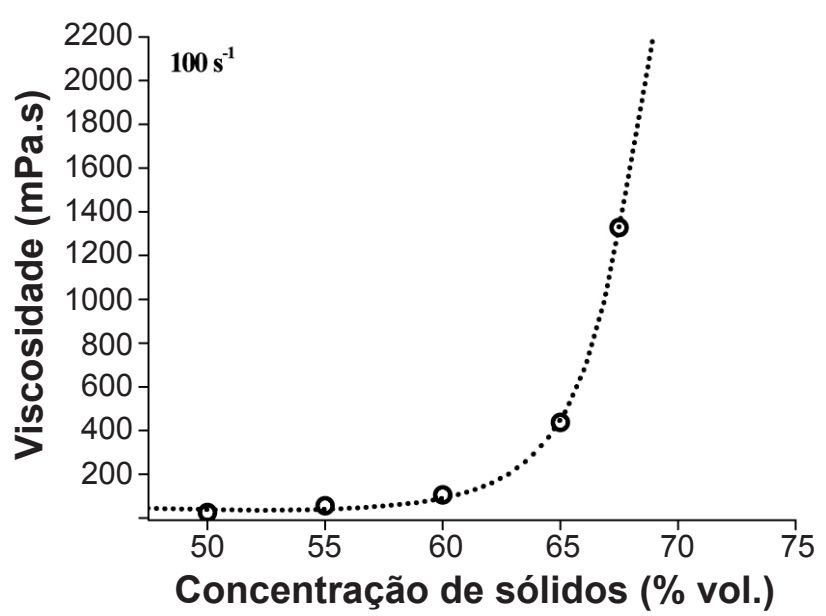

Figura 3: Curva de viscosidade de suspensões de alumina em função da concentração de sólidos a uma velocidade de cisalhamento constante de $100 \mathrm{~s}^{-1}$.

[Figure 3: Viscosity of alumina suspensions versus volume fraction of solids curve for a constant shear rate at $100 \mathrm{~s}^{-1}$.]

Às suspensões concentradas produzidas, adicionou-se o HPMC, e à medida que o teor do espessante aumentou as viscosidades das suspensões também apresentaram um acentuado aumento, dificultando a continuidade da homogeneização em agitador mecânico. Para garantir a homogeneidade das massas cerâmicas produzidas, passouse a utilizar um moinho de facas, semelhante a uma extrusora de rosca. O estudo no reômetro capilar fez-se considerando a força de atrito em função do deslocamento medida dentro do equipamento, conforme apresentado na Fig. 4, para uma velocidade constante.

O comportamento reológico das massas produzidas, com diferentes teores de HPMC, é apresentado na Fig. 5. 


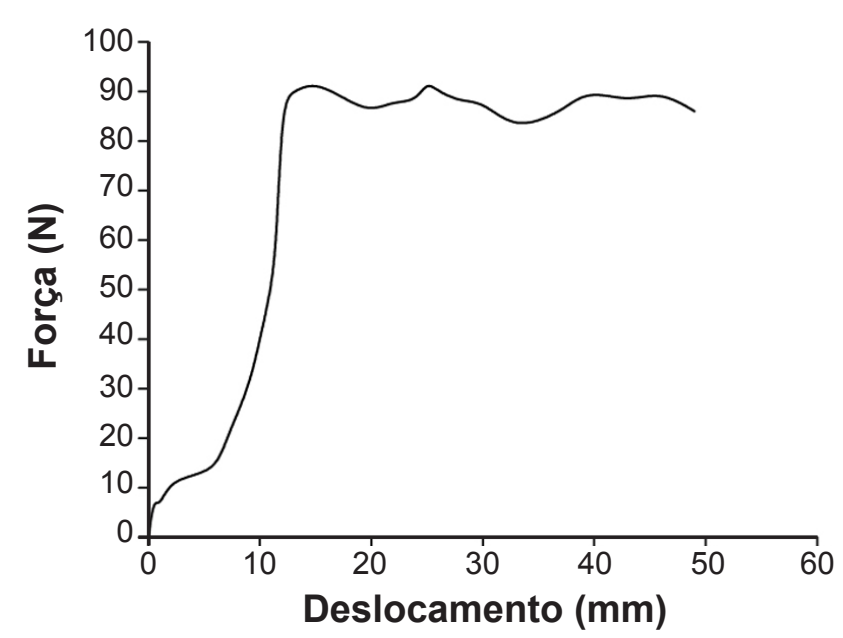

Figura 4: Curva de força em função do deslocamento. [Figure 4: Force versus displacement curve.]

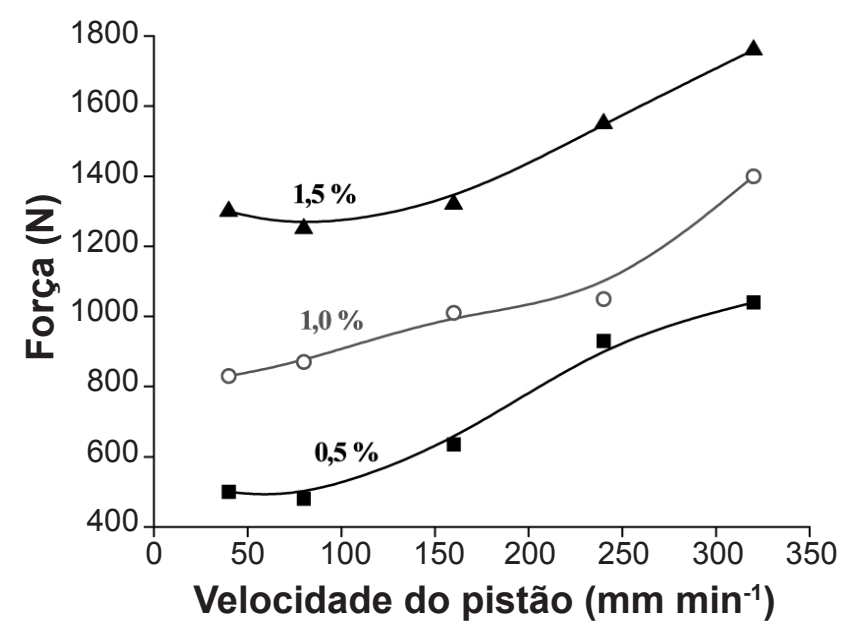

Figura 5: Curvas de força por velocidade de deslocamento, obtidas em reômetro capilar, para massas com diferentes conteúdos de HPMC.

[Figure 5: Displacement velocity versus force curves attained in capillary rheometer for different HPMC content pastes.]

Observa-se que, para uma mesma velocidade do pistão, o incremento na concentração do espessante, aumenta a força necessária ao fluxo da massa estudada, comportamento este, esperado. O aumento na velocidade do pistão, para uma mesma concentração de HPMC, promove um aumento na resistência ao deslocamento do pistão indicando comportamento fluidificante para as massas. Este comportamento, a fluidificação em função do cisalhamento, pode ser melhor observado na Fig. 6 onde, por meio dos resultados obtidos (força) e considerando as velocidades utilizadas, a geometria e as dimensões do equipamento utilizado [16], foi possível calcular as viscosidades para as diversas condições analisadas. É importante ressaltar, que os altos valores de viscosidade observados, são devidos às massas cerâmicas, não mais suspensões. Estes valores, desta forma, são compatíveis à condição que os materiais se encontram.

Após caracterização reológica das massas com diferentes

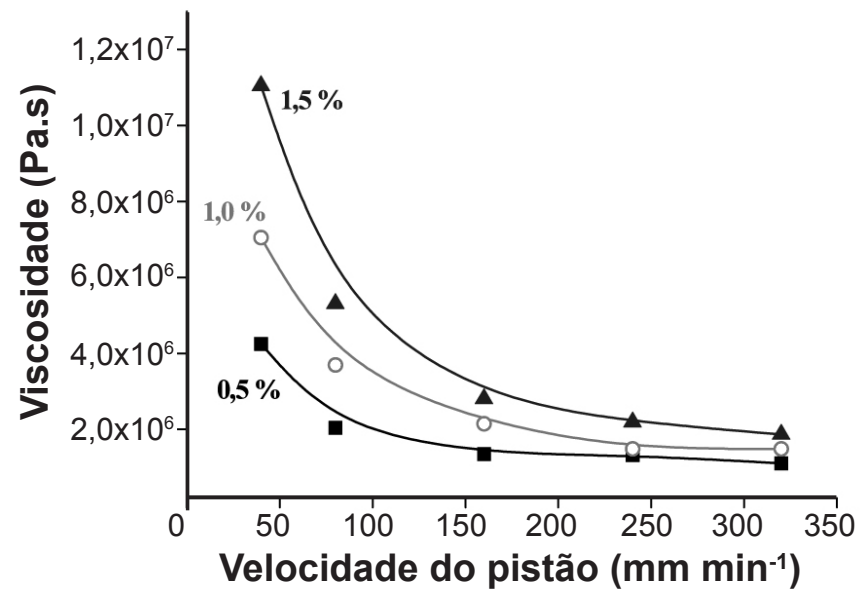

Figura 6: Curvas de viscosidade por velocidade de deslocamento, obtida em reômetro capilar, para massas com diferentes conteúdos de HPMC.

[Figure 6: Displacement velocity versus viscosity curves attained in capillary rheometer for different HPMC content pastes.]

conteúdos de HPMC, iniciou-se o estudo de conformação por rolos. Para esta etapa do trabalho, e considerando as diferentes espessuras e velocidades dos rolos da calandra, determinaram-se os parâmetros reológicos que influenciam a conformação dos substratos cerâmicos. Estes parâmetros são apresentados na Tabela I. Os parâmetros determinantes, considerados neste trabalho, para as melhores condições de conformação por calandragem são: as densidades relativas a verde e após sinterização, em relação à densidade teórica (Dt), e o módulo de resistência a flexão (MRF).

As massas plásticas com diferentes teores do espessante foram então conformadas por calandragem mantendo-se constantes, inicialmente, a rotação de $20 \mathrm{rpm}$ e distância entre rolos de $1,5 \mathrm{~mm}$. O melhor comportamento de

Tabela I - Parâmetros reológicos envolvidos na conformação por calandragem.

[Table I - Rheological parameters for calendering shaping.]

\begin{tabular}{cccc}
\hline $\begin{array}{c}\text { Rotação } \\
\text { dos rolos } \\
(\mathrm{rpm})\end{array}$ & $\begin{array}{c}\text { Velocidade } \\
\text { dos rolos } \\
\left(\mathrm{mm} \cdot \mathrm{s}^{-1}\right)\end{array}$ & $\begin{array}{c}\text { Abertura } \\
\text { entre } \\
\text { rolos }(\mathrm{mm})\end{array}$ & $\begin{array}{c}\text { Velocidade de } \\
\text { cisalhamento } \\
\text { para abertura } \\
\text { entre rolos }\left(\mathrm{s}^{-1}\right)\end{array}$ \\
\hline \multirow{2}{*}{5} & 10,5 & 1,5 & 14,0 \\
& & 3,0 & 7,0 \\
& & 4,0 & 5,2 \\
20 & 41,9 & 1,5 & 55,9 \\
& & 4,0 & 27,9 \\
& & 4,0 & 20,9 \\
& & 1,5 & 139,6 \\
& 104,7 & 3,0 & 69,8 \\
& & 4,0 & 52,4 \\
\hline
\end{tabular}


conformação do substrato foi o observado nas massas preparadas com $1,5 \% \mathrm{~m}$ do espessante/plastificante e assim, considerou-se este valor como otimizado. O resultado para esta etapa de conformação é mostrado na Tabela II. É possível observar o aumento da densidade a verde com o aumento da concentração do espessante/plastificante, porém, os valores das densidades relativas após sinterização estão muito próximos para todas as amostras. Pode-se notar também que o módulo de resistência a flexão aumenta à medida que a concentração de HPMC também aumenta. Este comportamento é atribuído a maior compacidade da massa com esta concentração $(1,5 \% \mathrm{~m})$, que garante maior empacotamento de partículas durante a aplicação da pressão pelos rolos, pois após conformação, os substratos mantiveram-se uniformes, com bom acabamento superficial e provavelmente com menor quantidade de defeitos na estrutura. Entretanto, ainda que o MRF tenha sido maior em relação às outras amostras, os aspectos estéticos, visualmente observados nos substratos após a conformação, foram determinantes para a escolha desta composição.

O próximo parâmetro avaliado na conformação por calandragem foi a variação na rotação dos rolos. Utilizandose a composição preparada com $1,5 \% \mathrm{~m}$ de HPMC e mantendo uma abertura de 1,5 mm entre rolos, observou-se a

Tabela II - Características dos substratos preparados por laminação a partir de massas de alumina com diferentes conteúdos de HMPC a $20 \mathrm{rpm}$ e espessura de $1,5 \mathrm{~mm}$, sinterizadas a $1600{ }^{\circ} \mathrm{C} / 1 \mathrm{~h}$.

[Table II - Characteristics of substrates shaped with different $H P M C$ contents at $20 \mathrm{rpm}$ and $1.5 \mathrm{~mm}$ height, sintered at $\left.1600{ }^{\circ} \mathrm{C} / 1 \mathrm{~h}.\right]$

\begin{tabular}{cccc}
\hline $\begin{array}{c}\text { Conteúdo de } \\
\text { HPMC } \\
(\% \mathrm{~m} .)\end{array}$ & $\begin{array}{c}\text { Densidade a } \\
\text { verde } \\
(\% \mathrm{Dt})\end{array}$ & $\begin{array}{c}\text { Densidade } \\
\text { sinterizada } \\
(\% \mathrm{Dt})\end{array}$ & $\begin{array}{c}\text { MRF } \\
(\mathrm{MPa})\end{array}$ \\
\hline 0,5 & $60,2 \pm 2,2$ & $99,4 \pm 0,2$ & $118 \pm 10$ \\
1,0 & $63,0 \pm 3,1$ & $99,4 \pm 0,2$ & $125 \pm 12$ \\
1,5 & $69,5 \pm 1,6$ & $99,5 \pm 0,3$ & $135 \pm 15$ \\
\hline
\end{tabular}

Tabela III - Características dos substratos conformados por laminação a diferentes rotações e sinterizadas a $1600^{\circ} \mathrm{C} / 1 \mathrm{~h}$. Composição com 1,5\%m de HPMC e substratos de espessura de $1,5 \mathrm{~mm}$.

[Table III - Characteristics of substrates shaped at different rotations with $1.5 \mathrm{wt} . \%$ HPMC content and $1.5 \mathrm{~mm}$ height, sintered at $\left.1600{ }^{\circ} \mathrm{C} / 1 \mathrm{~h}.\right]$

\begin{tabular}{cccc}
\hline $\begin{array}{c}\text { Rotação } \\
(\mathrm{rpm})\end{array}$ & $\begin{array}{c}\text { Densidade } \\
\text { a verde } \\
(\% \mathrm{Dt})\end{array}$ & $\begin{array}{c}\text { Densidade } \\
\text { sinterizada } \\
(\% \mathrm{Dt})\end{array}$ & $\begin{array}{c}\text { MRF } \\
(\mathrm{MPa})\end{array}$ \\
\hline 5 & $68,2 \pm 2,2$ & $99,6 \pm 0,2$ & $123 \pm 10$ \\
20 & $69,5 \pm 1,6$ & $99,5 \pm 0,3$ & $135 \pm 15$ \\
50 & $68,7 \pm 1,3$ & $99,4 \pm 0,3$ & $131 \pm 13$ \\
\hline
\end{tabular}

influencia da velocidade de rotação dos rolos em função das densidades a verde e sinterizada e na resistência mecânica. Os resultados desta análise, executados a 5, 20 e 50 rpm, são apresentados na Tabela III. Observa-se uma leve redução nos valores de densidade após a sinterização com o aumento da rotação, o que poderia sugerir que se aumentando a velocidade de rotação, aumenta-se o cisalhamento sobre as
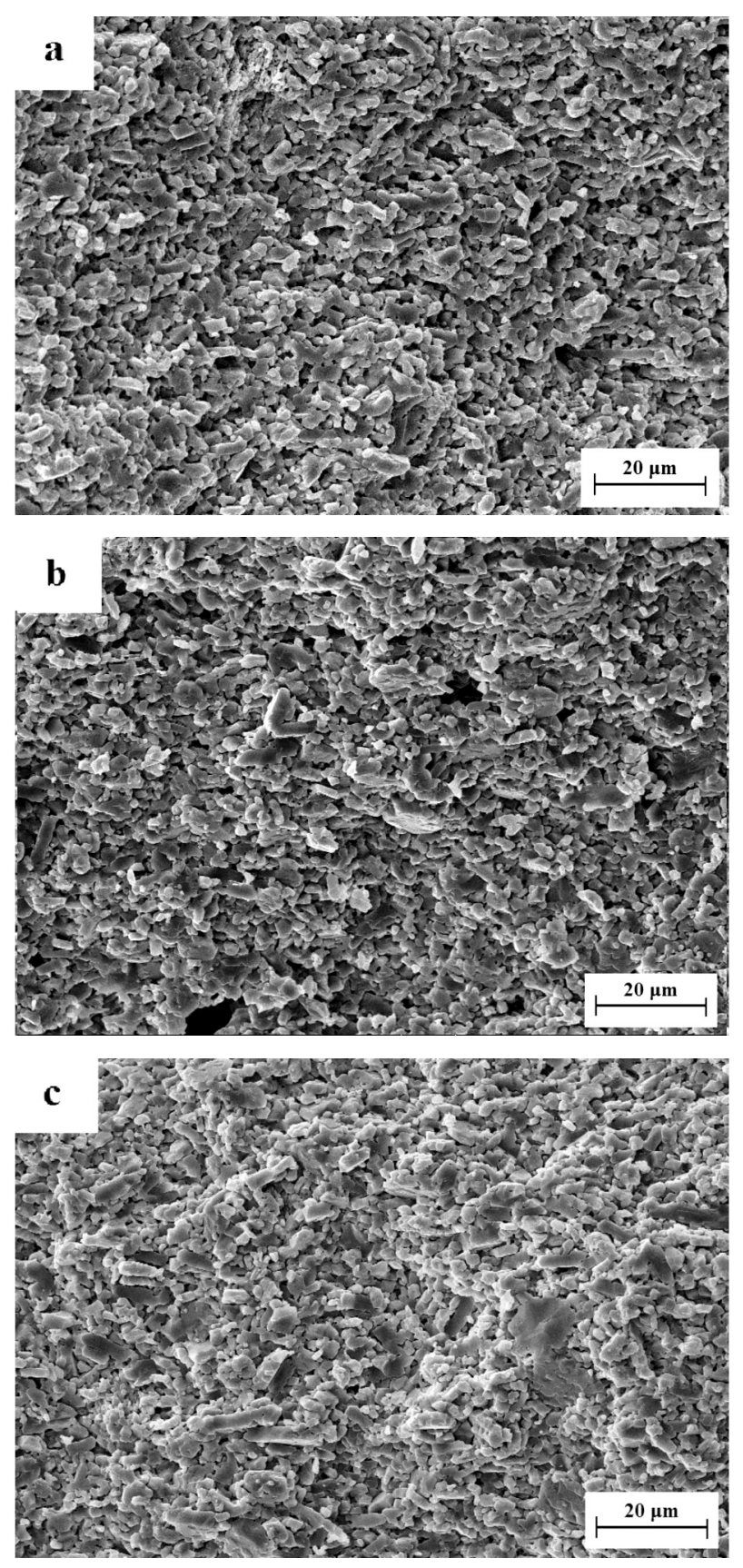

Figura 7: Micrografias obtidas por microscopia eletrônica de varredura das superfícies de fratura de substratos conformados com $1,5 \% \mathrm{~m}$. de HPMC a $20 \mathrm{rpm}$ e sinterizadas a $1600{ }^{\circ} \mathrm{C} / 1 \mathrm{~h}$ : (a) 1,5 ; (b) 3,0 e (c) 4,0 mm de espessura.

[Figure 7: SEM micrographs of fracture surfaces of substrates shaped with $1.5 \mathrm{wt} . \% \mathrm{HPMC}$ at $20 \mathrm{rpm}$ and sintered at $1600{ }^{\circ} \mathrm{C} / 1 \mathrm{~h}$ : (a) 1.5; (b) 3.0 and (c) $4.0 \mathrm{~mm}$ thickness.] 
massas alterando o empacotamento das partículas, entretanto, isso não procede. Os desvios nos valores de densidade mostram uma igualdade nos resultados e, além disso, quando se observa os valores das velocidades de cisalhamento nas diversas condições de conformação (Tabela I), e as densidades calculadas para as massas no reômetro capilar, conclui-se que a influência da velocidade exercida pelos rolos sobre as massas cerâmicas é mínima. Os resultados mostram valores muito próximos em todas as condições avaliadas. Para esta etapa de processo, considerando que os valores obtidos foram praticamente iguais, optou-se para continuidade do trabalho a rotação de $20 \mathrm{rpm}$ pois, em rotações menores a conformação dos substratos é lenta, causando muitas vezes ondulações superficiais, e em velocidades maiores, o controle da passagem das massas entre os rolos torna-se difícil.

Definidos os parâmetros de concentração de espessante/ plastificante $(1,5 \% \mathrm{~m})$ e da rotação entre rolos $(20 \mathrm{rpm})$, a próxima etapa versou na influência da espessura na conformação dos substratos de alumina. Considerou-se nesta etapa as aberturas entre rolos de 1,5, 3,0 e 4,0 mm. Na Tabela IV são apresentadas as características dos substratos produzidos, onde pode se observar, da mesma maneira que nas rotações estudadas, características praticamente iguais. $\mathrm{O}$ mesmo efeito das velocidades de cisalhamento (Tabela I) sobre as massas cerâmicas, observados quando se variou a rotação entre rolos, é válido nesta etapa. Os elevados valores de viscosidade das massas (Fig. 6) praticamente não são alterados pelas baixas velocidades de cisalhamento proporcionadas no processo de calandragem e assim, as únicas diferenças observadas são as diferentes espessuras. As micrografias das superfícies de fratura dos substratos conformados a diferentes espessuras com 1,5\%m. de HPMC a 20 rpm são apresentadas na Fig. 7. As microestruturas apresentam o mesmo aspecto, com uniformidade na distribuição de grãos e alguma porosidade para as três espessuras conformadas, ratificando as observações anteriores.

Tabela IV - Características dos substratos conformados com diferentes espessuras após sinterização a $1600{ }^{\circ} \mathrm{C} / 1 \mathrm{~h}$. Composição com $1,5 \%$ m. HPMC e velocidade dos rolos de laminação de $20 \mathrm{rpm}$.

[Table IV - Characteristics of substrates shaped at different heights at $20 \mathrm{rpm}$ with $1.5 \mathrm{wt} \%$ HPMC content, sintered at $\left.1600{ }^{\circ} \mathrm{C} / 1 \mathrm{~h}.\right]$

\begin{tabular}{cccc}
\hline $\begin{array}{c}\text { Espessura } \\
(\mathrm{mm})\end{array}$ & $\begin{array}{c}\text { Densidade } \\
\text { a verde } \\
(\% \mathrm{Dt})\end{array}$ & $\begin{array}{c}\text { Densidade } \\
\text { sinterizada } \\
(\% \mathrm{Dt})\end{array}$ & $\begin{array}{c}\text { MRF } \\
(\mathrm{MPa})\end{array}$ \\
\hline 1,5 & $69,5 \pm 1,6$ & $99,5 \pm 0,3$ & $135 \pm 15$ \\
3,0 & $63,0 \pm 3,1$ & $99,7 \pm 0,2$ & $124 \pm 10$ \\
4,0 & $69,5 \pm 1,6$ & $99,6 \pm 0,2$ & $121 \pm 11$ \\
\hline
\end{tabular}

\section{CONCLUSÕES}

A preparação de substratos cerâmicos plásticos densos, por meio de suspensões iniciais com alta concentração de sólidos, é possível com pequena concentração de aditivos $(\sim 1,5 \% \mathrm{~m})$. A utilização do dispersante comercial Viscocrete HE20 mostrou-se eficiente na estabilização de suspensões de alumina, em concentrações de $0,02 \%$, que é inferior a uma ordem de 10 em relação a concentração necessária com dispersantes tradicionalmente utilizados, como o ácido cítrico. Com a adição de apenas 1,5\%, em massa, de HPMC 3000 às suspensões concentradas de alumina ( $60 \%$ vol.), foi possível obter massas cerâmicas plásticas adequadas para conformação por rolos a frio (calandragem). As variáveis de processo avaliadas, velocidade de rotação e abertura entre rolos, devido aos altos valores de viscosidade das massas produzidas, não influenciam significativamente nas características finais dos substratos cerâmicos, e para todas as condições estudadas foi possível a obtenção de peças íntegras e densas.

\section{AGRADECIMENTOS}

À FAPESP pela bolsa de Pós-doutorado de Luiz Fernando Grespan Setz e à CAPES e ao CNPq pelas bolsas de doutorado de Arturo Rodrigo Ferreira Pardo e de Laís Koshimizu.

\section{REFERÊNCIAS}

[1] P. Souza Santos, "Ciência e Tecnologia de Argilas", 2a Ed, Vol. 1, Edgard Blücher Ltda., S. Paulo, SP (1989).

[2] J. S. Reed, "Principles of Ceramics Processing", $2^{\text {nd }}$ Ed., John Wiley \& Sons, Inc., New York, EUA (1995).

[3] J. J. Benbow, E. W. Oxley, J. Bridgwater, Chem. Eng. Sci. 42 (1987) 2151.

[4] M. J. Ribeiro, J. M. Ferreira, J. A. Labrincha, Ceram. Int. 31 (2005) 515.

[5] D. W. Richerson, "Modern Ceramic Engineering: Properties, Processing, and Use in Design", $2^{\text {nd }}$ Ed., Marcel Dekker, New York, EUA (1992).

[6] R. Moreno, "Reología de Suspensiones Cerámicas", Consejo Superior de Investigaciones Científicas, Madrid, Espanha (2005).

[7] R. W. Baker, "Membrane Technology and Applications", $2^{\text {nd }}$ Ed., John Wiley \& Sons Inc., Chichester, UK (2004).

[8] V. Boljanovic, "Metal Shaping Processes", $1^{\text {st }}$ Ed., Industrial Press, New York, EUA (2009).

[9] H. Tschätsch, "Metal Forming Practise: Processes Machines - Tools", ${ }^{\text {st }}$ Ed., Springer-Verlag, Berlin, Alemanha (2006).

[10] F. A. Mesquita, M. R. Morelli, J. Mater. Process. Tech. 143-144 (2003) 232.

[11] A. R. F. Pardo, M. R. Morelli, "Processamento Viscoplástico e Conformação Cerâmica por Rolos a Frio a Partir de Suspensões Concentradas de Alumina", Tese Dr., Departamento de Engenharia de Materiais, Universidade Federal de S. Carlos, S. Carlos, SP (2005).

[12] P. A. L. Fernandes, E. N. B. S. Júlio, P. M. N. Tiago, "Vigas de Grande Vão Pré-fabricadas em Betão de Alta Resistência Pré-esforçado: Viabilidade, Dimensionamento, 
Fabrico e Comportamento", Tese Dr., Departamento de Engenharia Civil, Universidade de Coimbra, Coimbra, Portugal (2005).

[13] P. C. Hidber, T. J. Graule, L. J. Gauckler, J. Am. Ceram. Soc. 79 (1996) 1857.

[14] J. N. Israelachvili, "Intermolecular and Surface Forces", $2^{\text {nd }}$ Ed., Academic Press, Oxford, UK (1992).

[15] D. J. Shaw, "Introduction to Colloid and Surface Chemistry", $4^{\text {th }}$ Ed., Butterworth-Heinemann, Oxford, UK (1992).

[16] F. Händle, "Extrusion in Ceramics", Springer-Verlag, Berlin, Germany (2007) 175.

(Rec. 28/10/2010, Rev. 16/02/2011, Ac. 11/03/2011) 